\title{
Insulin-like growth factor 2 mRNA-binding protein-3 as a marker for distinguishing between cutaneous squamous cell carcinoma and keratoacanthoma
}

\author{
AKIKO KANZAKI ${ }^{1,2}$, MITSUHIRO KUDO $^{1}$, SHIN-ICHI ANSAI $^{3}$, WEI-XIA PENG ${ }^{1}$, KOUSUKE ISHINO $^{1}$, \\ TETSUSHI YAMAMOTO ${ }^{4}$, RYUICHI WADA ${ }^{1}$, TAKENORI FUJII ${ }^{1}$, KIYOSHI TEDUKA ${ }^{1}$, KIYOKO KAWAHARA ${ }^{1}$, \\ YOKO KAWAMOTO ${ }^{1}$, TAEKO KITAMURA ${ }^{1}$, SEIJI KAWANA ${ }^{2}$, HIDEHISA SAEKI ${ }^{2}$ and ZENYA NAITO $^{1}$ \\ Departments of ${ }^{1}$ Integrated Diagnostic Pathology, ${ }^{2}$ Dermatology, Nippon Medical School; ${ }^{3}$ Department of Dermatology, \\ Nippon Medical School Musashikosugi Hospital, Tokyo; ${ }^{4}$ Faculty of Pharmacy, Kinki University, Osaka, Japan
}

Received October 29, 2015; Accepted December 8, 2015

DOI: 10.3892/ijo.2016.3323

\begin{abstract}
In the histopathological diagnosis of cutaneous tumors, the differential diagnosis of squamous cell carcinoma (SCC) with crateriform architecture and keratoacanthoma (KA) is often difficult so an accurate understanding of the biological features and the identification of reliable markers of SCC and KA are crucial issues. Insulin-like growth factor 2 mRNA-binding protein-3 (IGF2BP3, also known as IMP3) is thought of as a bona fide oncofetal protein, which is overexpressed and is involved in cell proliferation, migration, and invasion in several kinds of tumors. However, the role of IMP3 in cutaneous SCC and KA has not been well studied. Therefore, we focused on studying the biological functions of IMP3 in SCC and KA. In human skin SCC cell lines, HSC-1 and HSC-5, and the human keratinocyte cell line, HaCaT, IMP3 mRNA levels were significantly higher than that of normal human skin. The knockdown of IMP3 expression reduced the proliferation of $\mathrm{HSC}-1$, and significantly reduced invasion by HSC- 1 and HSC-5. In contrast, the knockdown of IMP3 did not significantly affect invasion by $\mathrm{HaCaT}$ cells. In immunohistochemical studies of SCC and KA tissues, the Ki-67 labeling index (LI) of the suprabasal cell layer was significantly higher in SCC, compared with KA tissues and the tumor-free margin (TFM) adjacent to SCC and KA. Most SCC tissues stained strongly positive for IMP3, but KA tissues and TFM were mostly negative for IMP3. The Ki-67 LI of the IMP3-positive group was significantly higher than that of the IMP3-negative group in the suprabasal cell layer of SCC. These results suggest that IMP3 plays an important
\end{abstract}

Correspondence to: Dr Mitsuhiro Kudo, Department of Integrated Diagnostic Pathology, Nippon Medical School, 1-25-16 Nezu, Bunkyo-ku, Tokyo 113-0031, Japan

E-mail:kudoh@nms.ac.jp

Key words: keratoacanthoma, squamous cell carcinoma, IMP3, invasion, migration role in proliferation and, more significantly, in the invasion of SCC, and may be a suitable marker for the histopathological diagnosis of SCC with a crateriform architecture and KA. Furthermore, IMP3 may potentially be a new therapeutic target for SCC.

\section{Introduction}

Cutaneous squamous cell carcinoma (SCC) is a metastatic cancer that originates from the squamous cells located in the suprabasal epidermis. Keratoacanthoma (KA) is characterized by a nodular exo-endophytic lesion with a keratin-filled crater; at a well-developed stage, the large, proliferating, pale pink cells have a glassy appearance $(1,2)$. KA was originally believed to be a benign neoplasm that showed complete resolution within a few months. However, Hodak et al asserted that KA should be classified as a subtype of SCC with a low-grade malignancy (3). Weedon et al regarded KA as a type of benign squamous proliferation that can show malignant transformation into SCC (4). Kossard et al proposed follicular SCC and infudibular SCC, a new variant of SCC, and these variants may refine the classification of KA (5). Misago et al considered these two variants of SCC to be similar and to represent the same neoplastic disease; also, that SCC with follicular differentiation was clinicopathologically distinct from KA (6). These studies by others indicate that during the histopathological diagnosis of a cutaneous tumor, the differential diagnosis of SCC with crateriform architecture and KA is often difficult, and a reliable marker to differentiate these pathological lesions has not been found.

The insulin-like growth factor 2 (IGF2) mRNA-binding protein (IMP) family consists of IMP1, IMP2, and IMP3. IMP3 is also known as L523S and K-homology $(\mathrm{KH})$ domaincontaining protein overexpressed in cancer (KOC) (7-9). IMP3 binds to and regulates IGF-2 transcripts, and is involved in the posttranscriptional regulation of cell proliferation during embryogenesis (8). The expression of IMP3 in normal tissues such as placenta, ovary, testis, internal root sheath of hair follicles, pituitary gland, and lymph node germinal centers has been demonstrated $(7,8,10-13)$. Liao et al described how 
IMP3 was a translational activator of IGF-2 leader-3 mRNA and promoted cell proliferation by inducing the translation of IGF-2 mRNA in human K562 leukemia cells (14). IMP3 overexpression has been demonstrated in various tumors, such as squamous cell carcinoma, melanoma and lung cancer (15-23).

In cutaneous cancer, it was claimed that IMP3 was a diagnostic clue to cutaneous melanocytic neoplasms because of its expression in malignant melanomas, but not in benign melanocytic nevi, even when dysplastic features were present $(17,19)$. Recently, Sheen et al confirmed that IMP3 expression was a poor prognostic factor in melanomas, especially acral lentiginous melanoma (ALM), and promoted migration and invasion of melanoma cells (18). Moreover, IMP3 was helpful in distinguishing benign intranodal nevi from metastatic melanoma in sentinel lymph node biopsy specimens (20). Soddu et al suggested IMP3 may be suitable for a differential diagnosis between KA and SCC based on IMP3 immunohistochemical findings (24). However, understanding the role of IMP3 in cutaneous SCC and KA using cell and molecular biological approaches has not been well studied. In this study, we confirmed that IMP3 expression promoted cell proliferation, migration and invasion in SCC cell lines using siRNA. In addition, Ki67 labeling indexes (LI) and IMP3 staining patterns in SCC and KA tissues were also examined.

\section{Materials and methods}

Cell culture. Human SCC cell lines (HSC-1, HSC-5) $(25,26)$ were obtained from the Japanese Collection of Research Bioresources (Osaka, Japan), and the immortalized human keratinocyte cell line, HaCaT, was purchased from CLS Cell Lines Service GmbH (Eppelheim, Germany). HSC-1 and HSC-5 cells were cultured in RPMI-1640 (Gibco, Grand island, NY, USA) medium supplemented with $10 \%$ heat-inactivated fetal bovine serum (FBS; Nichirei Biosciences Inc., Tokyo Japan), and HaCaT cells were cultivated in DMEM medium supplemented with $10 \% \mathrm{FBS}$, at $37^{\circ} \mathrm{C}$ under a humidified $5 \%$ $\mathrm{CO}_{2}$ atmosphere.

Reverse transcription-quantitative polymerase chain reaction $(R T-q P C R)$. A total of $2.5 \times 10^{5}$ cells were seeded in $60-\mathrm{mm}$ dishes and cultured for $48 \mathrm{~h}$. Total RNA was extracted by Fast Pure RNA kit, and $1 \mu \mathrm{g}$ of total RNA was used for reverse transcription using a High Capacity cDNA Reverse Transcription Kit following the manufacturer's protocol (Applied Biosystems, Foster City, CA, USA). We performed RT-qPCR for IMP3 and 18S rRNA (as an internal standard) using a StepOnePlus Real-Time PCR system (Life Technologies, Carlsbad, CA, USA) with specific primers (18S: Hs 03928990_g1, IMP3: Hs 00559907_g1, Applied Biosystems) and a TaqMan probe (Applied Biosystems). Cycling conditions were as follows: $20 \mathrm{sec}$ at $95^{\circ} \mathrm{C}$, and then 40 cycles of $1 \mathrm{sec}$ at $95^{\circ} \mathrm{C}$ and $20 \mathrm{sec}$ at $60^{\circ} \mathrm{C}$. RT-qPCR results are expressed as the ratio of target mRNA to 18S rRNA. Gene expression levels were measured in triplicate. MVP human skin total RNA (Agilent Technologies, Santa Clara, CA, USA) was used for comparing the IMP3 mRNA expression level of normal skin and cell lines.

Western blotting. To extract total protein for determining IMP3 protein expression levels, cells were lysed in urea/thiourea buffer containing $7 \mathrm{M}$ urea, $2 \mathrm{M}$ thiourea, 3\% 3-[3-(cholamidopropyl)dimethylammonio]-1-propanesulphonate (CHAPS), and $1 \%$ Triton $\mathrm{X}-100$. Cell lysates were centrifuged at $3000 \mathrm{rpm}$ for $10 \mathrm{~min}$ at $4^{\circ} \mathrm{C}$ and the supernatants were centrifuged at $15,000 \mathrm{x} \mathrm{g}$ for $30 \mathrm{~min}$ at $4^{\circ} \mathrm{C}$. The resulting supernatants were collected as a cell extract, and the protein concentration was measured using the Bradford method. An equal amount of protein for each cell extract was loaded and separated by $10 \%$ sodium dodecyl sulfate-polyacrylamide gel electrophoresis (SDS-PAGE) and then electrophorectically blotted onto a polyvinylidene difluoride (PVDF) membrane (Immobilon-P, EMD Millipore, Billerica, MA, USA). Blots were blocked for $1 \mathrm{~h}$ with 5\% skim milk in Tris-buffered saline (TBS) containing $0.2 \mathrm{M}$ Tris- $\mathrm{HCl}, 150 \mathrm{mM} \mathrm{NaCl}$, and $0.01 \%$ Tween-20, and then incubated with anti-human IMP3 monoclonal mouse antibody (1:1000; Dako, M3626, clone: 69.1, Glostrup, Denmark) or anti$\beta$-actin monoclonal mouse antibody (1:10,000; Sigma-Aldrich, clone: AC-74, St. Louis, MO, USA), followed by overnight incubation at $4^{\circ} \mathrm{C}$.

After 30 min washing in TBS with $0.01 \%$ Triton $\mathrm{X}-100$, the blots were incubated with a 1:10,000 dilution of horseradish peroxidase-conjugated secondary antibody (A106PU, American Qualex Antibodies, San Clemente, CA, USA) for $1 \mathrm{~h}$ at room temperature (RT). Immunoreactive products were visualized using a SuperSignal West Dura Extended Duration Substrate (Thermo Fisher Scientific, Waltham, MA, USA) for IMP3, and a Super Signal West Pico Chemiluminescence substrate (Thermo Fisher Scientific) for $\beta$-actin. Experiments were performed in triplicate.

Knockdown of IMP3 expression in HSC-1, HSC-5 and HaCaT cells. Short-interfering RNA (siRNA) was used to determine the influence of IMP3 in cellular kinetics such as cell proliferation, migration, and invasion. HSC-1, HSC-5 and HaCaT cells were transfected by Lipofectamine ${ }^{\circledR}$ RNAiMAX Reagent (Invitrogen/ThermoFisher Scientific, Carlsbad, CA, USA) with $5 \mathrm{nM}$ of silencer pre-designed siRNA for the knockdown of IMP3 expression (siIMP3; \#4392420, Ambion/ThermoFisher Scientific), or $5 \mathrm{nM}$ of silencer negative control siRNA for the negative control (siCtrl; \#4390844, Ambion) according to the manufacturer's protocol. Total RNA and total protein were extracted, respectively, after 48 and $72 \mathrm{~h}$ of siRNA treatment. Experiments were performed in triplicate.

Cell proliferation assay. Cells were seeded in 96-well plates at a density of 5,000 cells per well, followed by culturing at $37^{\circ} \mathrm{C}$ in a humidified $5 \% \mathrm{CO}_{2}$ atmosphere after siRNA treatments: siRNA for IMP3 (siIMP3) and siRNA for negative control (siCtrl), as described above. After 24, 48, 72 and $96 \mathrm{~h}$ growth, cells were incubated with WST- 8 cell counting reagent (Dojindo, Kumamoto, Japan) for $2 \mathrm{~h}$ at $37^{\circ} \mathrm{C}$. The optical density of the culture solution in each well were determined at $450 \mathrm{~nm}$ using an ELISA plate reader (model 680, BioRad Laboratories, Hercules, CA, USA). Experiments were performed in triplicate.

Cell migration and invasion assays. In vitro migration and invasion assays were carried out using BioCoat control inserts and BioCoat Matrigel-coated inserts with BioCoat chambers (BD Bioscience, Franklin Lakes, NJ, USA), respectively. 
A

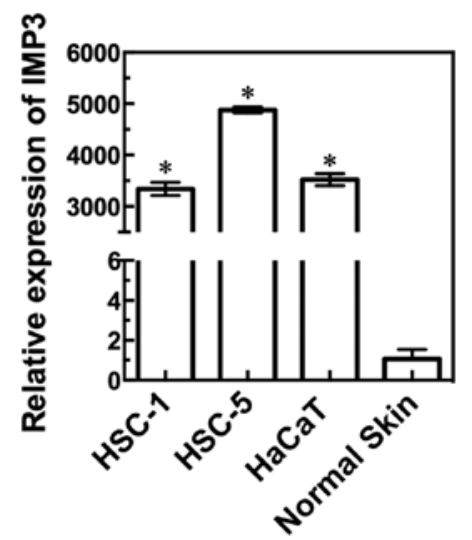

B
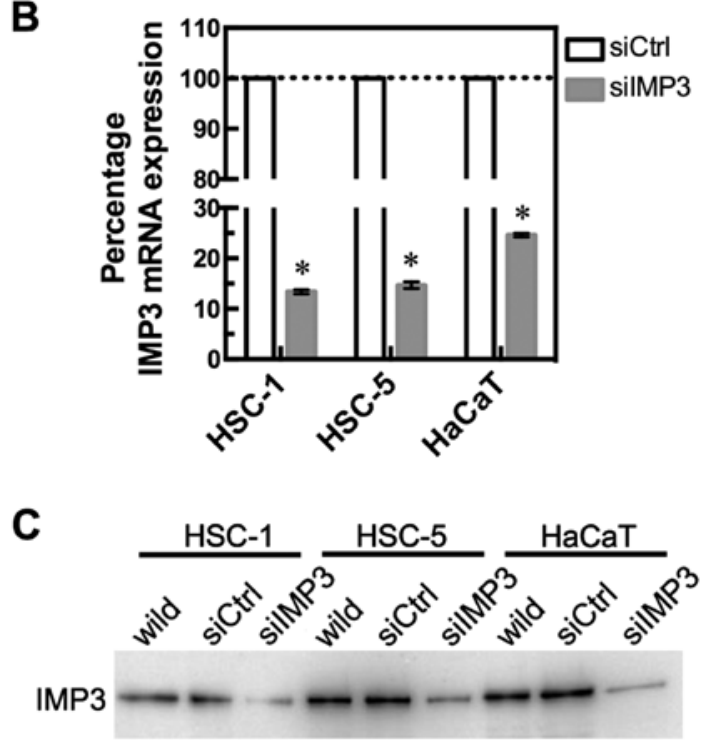

$\beta$-actin

Figure 1. IMP3 expression in HSC-1, HSC-5 and HaCaT cells, and normal human skin, and knockdown of IMP3 using siRNA. IMP3 mRNA expression was observed in HSC-1, HSC-5 and HaCaT cell lines and normal skin by RT-qPCR analysis. The expression of IMP3 relative to that in normal skin is shown (A). Knockout of IMP3 mRNA by siRNA in HSC-1, HSC-5 and $\mathrm{HaCaT}$ cell lines. IMP3 mRNA is expressed as a percentage of that in siRNA control transfected cells (100\%) (B). For (A) and (B), results are expressed as mean $\pm 95 \%$ confidence interval. ${ }^{*} \mathrm{P}<0.0001$, compared to normal skin (A) and si-control (siCtrl)-transfected cells (B). Western blot of IMP3 protein expression after treatment with IMP3 siRNA in HSC-1, HSC-5 and HaCaT cell lines. IMP3 protein levels in untransfected wild-type, si control (siCtrl) or siIMP3 RNA-transfected cells are shown (C).

After siRNA treatment, cells were harvested and suspended in serum-free RPMI-1640 for HSC-1 and HSC-5 cells, and DMEM for HaCaT cells. The cells were applied to the surface of control or Matrigel-coated inserts at a density of $1 \times 10^{5}$ cells per insert, and culture medium with $10 \%$ FBS was added to the lower chamber to serve as chemoattractant. After $24 \mathrm{~h}$ incubation for HSC-1 and HSC-5 cells, and $36 \mathrm{~h}$ of incubation for $\mathrm{HaCaT}$ cells at $37^{\circ} \mathrm{C}$ in a humidified $5 \% \mathrm{CO}_{2}$ atmosphere, migrating and invading cells were stained with Diff-Quick stain $^{\mathrm{TM}}$ (Sysmex Corp., Kobe, Japan). Stained cells on the outer surface in each of five fields per inserts were counted using bright field microscopy (Olympus, Tokyo, Japan) and a 20x objective. Experiments were performed in triplicate.
Formalin-fixed paraffin-embedded (FFPE) tissue samples. A total of eight cases of KA and 15 cases of SCC, which were classified on a conventional or actinic keratosis basis between 2009 to 2015 and procured from the archives at Nippon Medical School from 2009 to 2015; six were male and two were female for KA, and 15 were male and five were female for SCC. The mean age was 61.75 (38-75) years for KA and 78.4 (53-92) years for SCC. A total of eight KA were excised from face (4/8), trunk (3/8) and extremity (1/8). A total of $15 \mathrm{SCC}$ were excised from face (10/15), head (3/15) and extremity (2/15). The mean of tumor size of KA and SCC were, respectively, $14.14 \mathrm{~mm}$ (ranged from 9 to $21 \mathrm{~mm}$ ) and $18.43 \mathrm{~mm}$ (ranged from 6 to $49 \mathrm{~mm}$ ). All 23 patients were stage I or II classified in the UICC-TNM staging system 7 without recurrent and metastatic lesions. KA tissue samples showed a characteristic architectural pattern (an exo-endophytic lesion with a central keratotic horn), with the involvement of unclear epithelial lips on both sides by the lesions themselves. A lobule of tumor consisted of large pale, pink cells with a glassy appearance and without nuclear atypia. SCC tissue samples showed elevated lesions with hyperkeratosis and acanthosis. The neoplastic lobules consisted of squamoid cells, which showed nuclear atypia in the dermis. This study was carried out in accordance with the principles embodied in the Declaration of Helsinki, 2013, and the Japanese Society of Pathology Ethics Committee. Informed consent for the use of skin tissues was obtained from all the patients.

Immunostaining and scoring. Tissue sections from a total of eight cases of KA and 15 cases of SCC were used for immunostaining. After deparaffinization, sections were pretreated in an autoclave at $121^{\circ} \mathrm{C}$ for $15 \mathrm{~min}$ in EDTA (pH 8.0) for IMP-3 staining, and $10 \mathrm{mM}$ citrate buffer ( $\mathrm{pH} \mathrm{6.0)}$ for Ki-67 staining. Endogenous peroxidase was blocked using $0.3 \%$ hydrogen peroxide in methanol for $30 \mathrm{~min}$. The sections were then incubated with anti-human IMP3 monoclonal mouse antibody (1:200, Dako, clone: 69.1, Tokyo, Japan) and anti-Ki-67 antibody (1:100, Dako, clone: MIB-1) in phosphate-buffered saline containing $1 \%$ bovine serum albumin at $4^{\circ} \mathrm{C}$ overnight. The sections were further incubated with Simple Stain MAX-PO (M; Nichirei Biosciences Inc.) for $30 \mathrm{~min}$ and peroxidase activity was visualized by diaminobenzidine. The sections were then counterstained with Mayer's hematoxylin. Semi-quantitative measurements were used for comparing IMP3 expression levels and the Ki-67 LI of KA and SCC tissue sections as described below. A Ki-67 LI was determined by selecting five equivalent fields in KA and SCC specimens as a percentage of Ki-67-positive cells. In addition, the Ki-67 LI of the tumorfree margin (TFM) adjacent to SCC and KA tissues was determined. To determine the localization and expression level of IMP3-positive cells in KA and SCC sections, the intensity and percentage of IMP3-positive cells were determined using the $\mathrm{H}$-score method (27). $\mathrm{H}$-score formula $=3 \mathrm{x}$ percentage of strongly staining cytoplasm $+2 \mathrm{x}$ percentage of moderately staining cytoplasm + percentage of weakly staining cytoplasm. The Ki-67 LI and H-score in each basal cell layer and suprabasal cell layer were determined. The suprabasal cell layer was prescribed by the cells located $1 \mathrm{~mm}$ from squamous metaplasia in SCC specimens. Ki-67 LI and H-scores were 

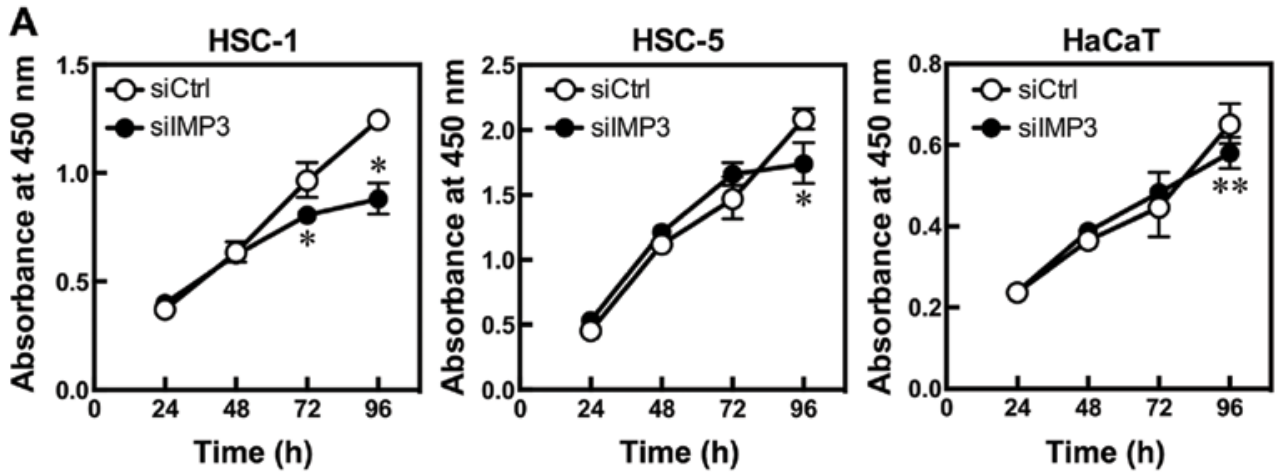

B
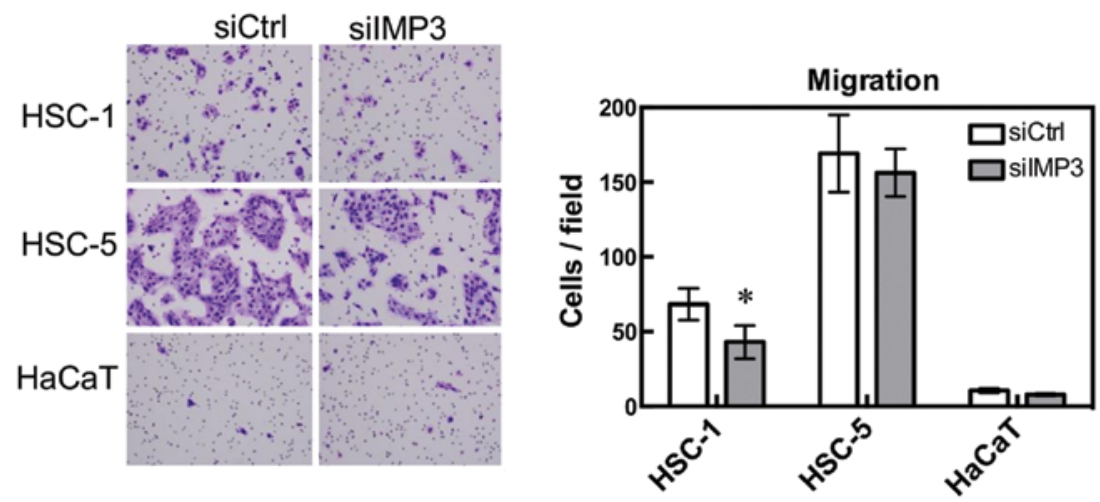

C
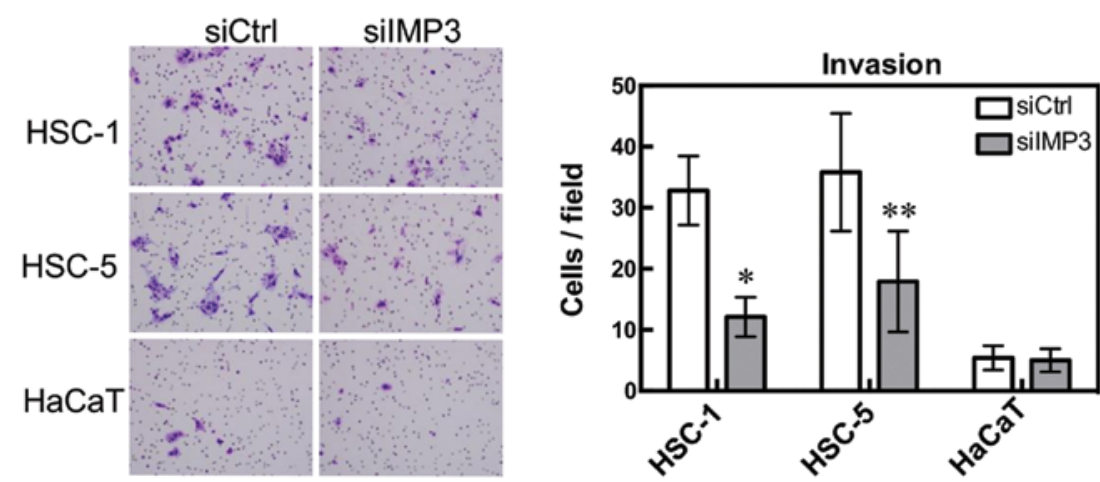

Figure 2. IMP3 is associated with cell proliferation, migration and invasion. HSC-1, HSC-5 and HaCaT cells were transfected with IMP3 siRNA and cell proliferation, migration and invasion were measured. Cell proliferation was determined by WST-8 cell counting reagent. HSC-1: "P<0.0001 for 72 and $96 \mathrm{~h}$, Sidak's multiple comparison test, and two-way ANOVA; and HSC-5: ${ }^{*} \mathrm{P}<0.0001$ for $96 \mathrm{~h}$; HaCaT: ${ }^{* *} \mathrm{P}=0.0008$ for $96 \mathrm{~h}$, Sidak's multiple comparison test, compared with si-control (siCtrl)-transfected cells (A). Cell migration was measured using BioCoat chambers. HSC-1: "P=0.0010, Mann-Whitney U-test, compared with siCtrl-transfected cells. HSC-5 and HaCaT cells were not affected (B). Cell invasion was determined using Matrigel-coated inserts in Boyden chambers. HSC-1: "P<0.0001; HSC-5: *" $\mathrm{P}=0.0063$, Mann-Whitney U-test compared with siCtrl-transfected cells (C). Results are expressed as mean $\pm 95 \%$ confidence interval. (B and C) Diff-Quick-stained cells. Magnification, x200.

evaluated by two investigators (A.K. and M.K.) in a blinded manner.

Statistical analysis. All data are shown using a two-sided 95\% confidence interval. Statistical comparisons between and among the groups were made using two-way ANOVA, Sidak's multiple comparison test, or a Mann-Whitney U-test. A value of $\mathrm{P}<0.05$ was considered significant. All statistical analyses were performed using GraphPad Prism version 5 (GraphPad Software, La Jolla, CA, USA).

\section{Results}

IMP3 expression in SCC cell lines and HaCaT cells. We confirmed IMP3 expression levels in two SCC cell lines, HSC-1 and HSC-5, and $\mathrm{HaCaT}$ cells by RT-qPCR (Fig. 1A). HSC-1, HSC-5, and HaCaT cells showed significantly higher levels of IMP3 mRNA, compared with normal skin (HSC-1: P<0.0001, HSC-5: $\mathrm{P}<0.0001, \mathrm{HaCaT}: \mathrm{P}<0.0001$, Mann-Whitney U-test, Fig. 1A). To examine the effects of IMP3 inhibition, we transfected IMP3 siRNA (siIMP3) into HSC-1, HSC-5, and HaCaT cells. RT-qPCR revealed that IMP3 mRNA expression was significantly decreased in silMP3 cells of these three cell lines (Fig. 1B). Thus, siIMP3-transfected cells were used in the following experiments to examine the inhibitory effects of IMP3.

IMP3 protein expression in siIMP3-transfected SCC and $\mathrm{HaCaT}$ cells. Western blot analysis revealed that the expression level of the $70 \mathrm{kDa}$ IMP3 protein was also downregulated 

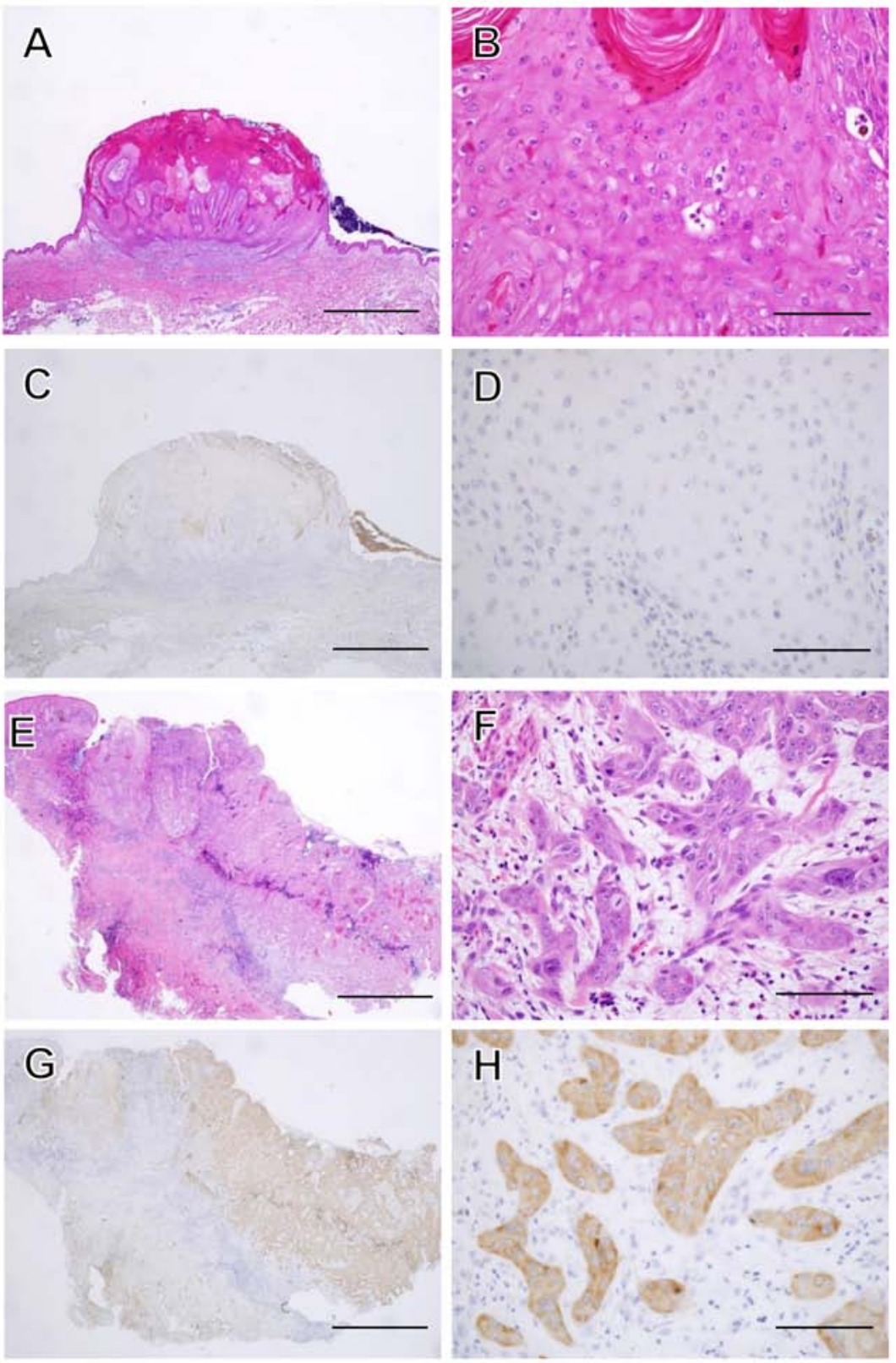

Figure 3. Histopathological features of KA and SCC. A magnified histological section showing a characteristic architectural pattern (an exo-endophytic lesion with a central keratotic horn) with the involvement of unclear epithelial lips on both sides by the lesions themselves (A). A close-up view of the KA of a lobule made up of large, pale pink cells with a glassy appearance without nuclear atypia (B). IMP3 was not expressed in the KA at all (C and D). A magnified histological section showed an elevated lesion with hyperkeratosis and acanthosis (E). In a close-up view of a SCC section, the neoplastic lobules consisted of squamoid cells, which showed nuclear atypia in the dermis (F). IMP3 was expressed diffusely in the neoplastic lobules of SCC (G and H). Hematoxylin and eosin (H\&E) staining was used (A, B, E and F). (C, D, G and H) Sections were stained with anti-IMP3 antibody and peroxidase activity visualized by diaminobenzidine; sections were counterstained with Mayer's hematoxylin. The scale bar is $2 \mathrm{~mm}(\mathrm{~A}, \mathrm{C}, \mathrm{E}$ and $\mathrm{G})$, and $100 \mu \mathrm{m}$ (B, D, F and $\mathrm{H})$.

in siIMP3-transfected HSC-1, HSC-5, and HaCaT cells compared to control siRNA (siCtrl)-transfected cells (Fig. 1C).

IMP3 siRNA downregulated SCC and HaCaT cell proliferation. To study the effects of reduced IMP3 expression on cell proliferation, siIMP3 and siCtrl-transfected cell lines were compared using WST- 8 cell counting reagent. The cell proliferation of siIMP3-transfected HSC-1 cells was significantly decreased at 72 and $96 \mathrm{~h}(\mathrm{P}<0.0001$ for both, Sidak's multiple comparison test, two-way ANOVA; Fig. 2A HSC-1) compared with siCtrl-transfected cells. The cell proliferation of siIMP3-transfected HSC-5 and HaCaT cells also tended to be significantly decreased at 96 h (HSC-5: P<0.0001; HaCaT: $\mathrm{P}=0.0008$, Sidak's multiple comparison test; Fig. 2A).

IMP3 siRNA downregulated SCC cell migration and invasion. To clarify the effect of IMP3 on cell migration and invasion, we used a modified Boyden chamber. The number of cells that migrated from the inside to the outside of the chamber was significantly decreased in siIMP3-transfected HSC-1 cells (HSC-1: $\mathrm{P}=0.0010$, Mann-Whitney U-test, Fig. 2B). The invasion assay using Matrigel-coated Boyden chambers showed that the number of invading cells decreased significantly for siIMP3-transfected HSC-1 and HSC-5 cells (HSC-1: $\mathrm{P}<0.0001$, HSC-5: $\mathrm{P}=0.0063$, Mann-Whitney U-test, Fig. 2C). 

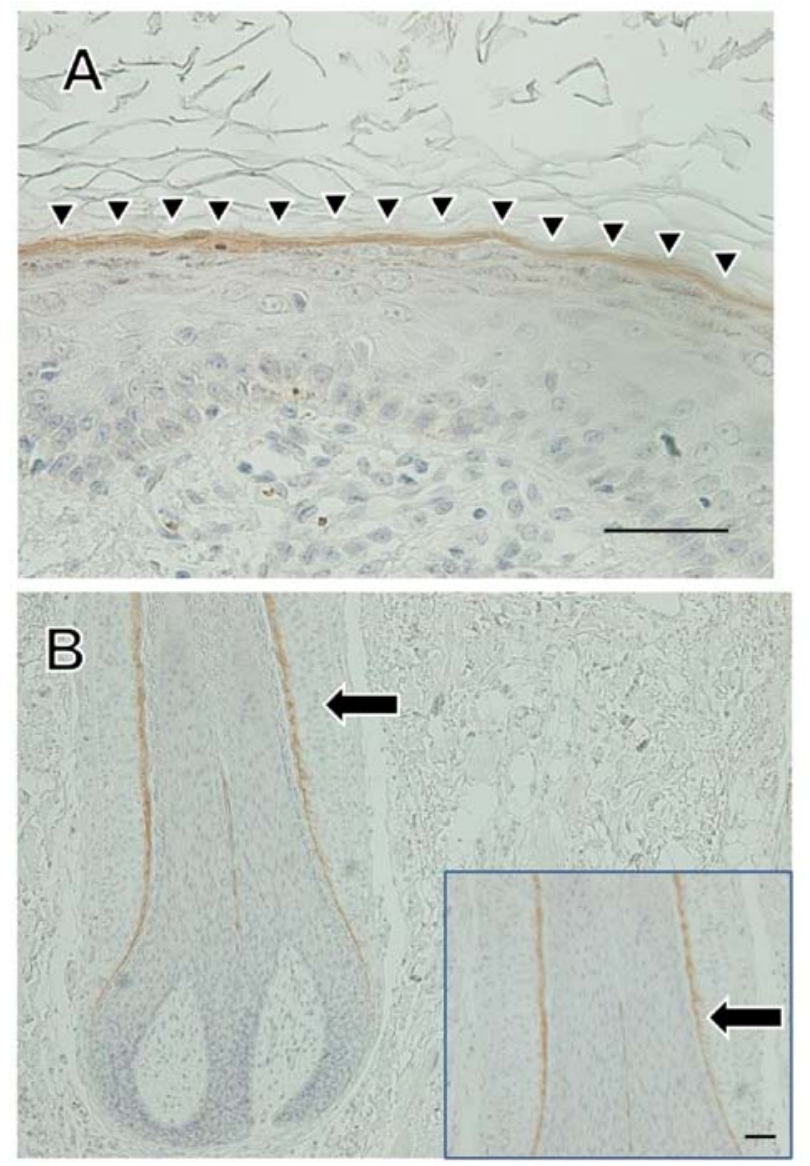

Figure 4. Immunohistochemical staining for IMP3 in normal skin. Immunohistochemical staining for IMP3 in normal skin sections was observed. IMP3 staining was observed in the granular cell layer (A, arrow heads), and in the internal root sheath of hair follicles (B, arrow). Scale bars: $50 \mu \mathrm{m}$.

HaCaT cells were not affected by IMP3 knockdown in either the cell migration or invasion assay.

Immunohistochemical analysis of IMP3 and Ki-67. We performed immunohistochemical staining of IMP3 and Ki-67 in SCC and KA tissues (Figs. 3, 5 and 6). IMP3 expression was not seen in normal skin except in the internal root sheath and granular layer (Fig. 4). A total of ten cases of SCC $(66.7 \%)$ were positive for IMP3 and, in contrast, all KA cases were negative for IMP3. IMP-3 expression was assessed as an $\mathrm{H}$-score (Fig. 5A). The $\mathrm{H}$-score for SCC tissues was significantly higher than that for KA tissues $(\mathrm{P}=0.0003$, Mann-Whitney U-test: Fig. 5B).

The Ki-67 LI of the suprabasal cell layer of SCC tissues was higher than that of KA and TFM tissues $(\mathrm{P}<0.0001$, Mann-Whitney U-test: Fig. 6B). The Ki-67 LI for the basal cell layer of SCC was higher than that for the suprabasal cell layers of SCC ( $\mathrm{P}=0.0445$, Mann-Whitney U-test; Fig. 6B) and for the basal cell layers of KA $(\mathrm{P}=0.8428$, Mann-Whitney U-test; Fig. 6B). The Ki-67 LI for the basal cell layer of KA was higher than that for the suprabasal cell layer of KA and for the basal cell layer of TFM $(\mathrm{P}<0.0001$, Mann-Whitney U-test; Fig. 6B).

To examine the relationship between Ki-67 and IMP3, we divided SCC cases into two groups, an IMP3-positive

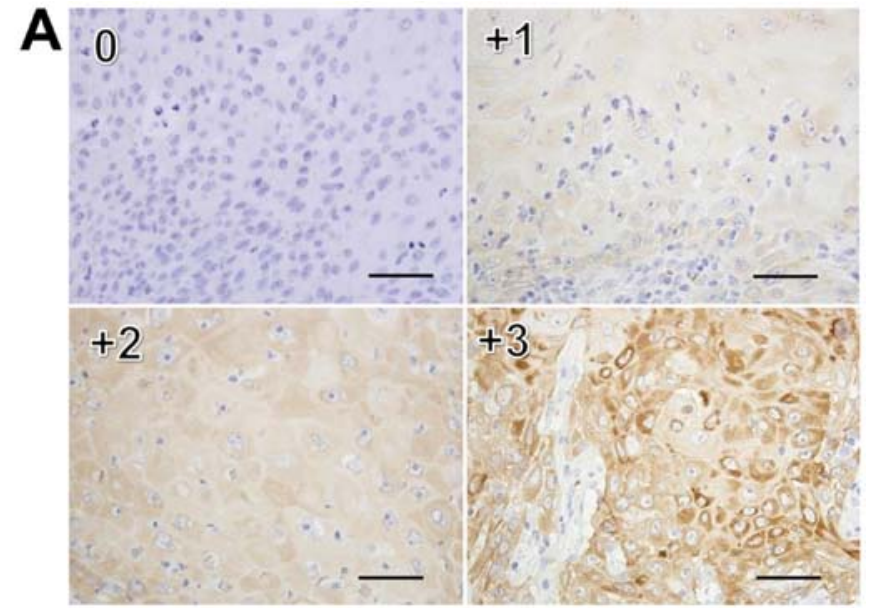

B

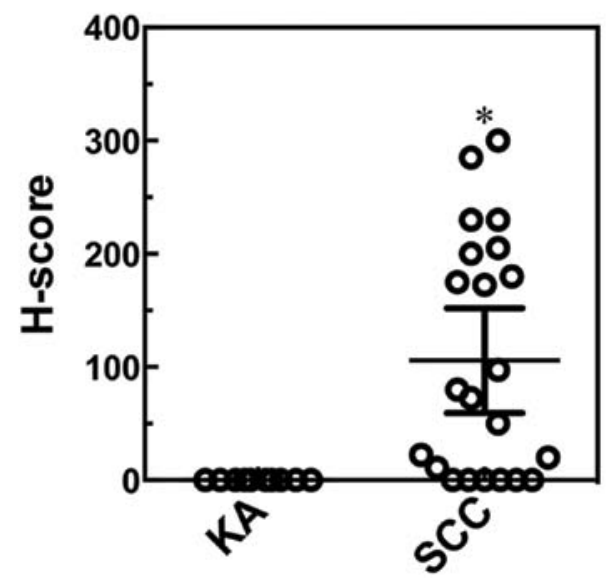

Figure 5. Immunohistochemical scoring system (H-score) for IMP3. Sections were stained with anti-IMP3 and peroxidase activity visualized by diaminobenzidine; sections were counterstained with Mayer's hematoxylin. Staining intensities for IMP3 are shown above $(0,+1,+2,+3)$, with an example each of a membranous and cytoplasmic staining pattern. Scale bars: $50 \mu \mathrm{m}$. Immunohistochemical scoring system (H-score) values for IMP3 in SCC and KA tissues. Results are expressed as mean $\pm 95 \%$ confidence interval. ${ }^{*} \mathrm{P}=0.0003$, Mann-Whitney U-test compared with KA (B).

group and an IMP3-negative group. We examined, in detail, the Ki-67 LI of basal and suprabasal cell layers in SCC. We found there was no significant difference in the Ki-67 LI of the basal cell layer between the IMP3-positive and -negative tumor groups ( $\mathrm{P}=0.4465$, Mann-Whitney U-test; Fig. 7A). However, the IMP3-positive group tended to show a high $\mathrm{Ki}-67 \mathrm{LI}$ for the suprabasal cell layer $(\mathrm{P}=0.0011$, MannWhitney U-test; Fig. 7B).

\section{Discussion}

IMP3 is thought of as a bona fide oncofetal protein that is overexpressed and is involved in cell proliferation, migration, and invasion in several kinds of tumors $(18,23,28,29)$. In regard to skin squamous neoplasms, the role of IMP3 in cutaneous SCC, using various cell biological and molecular biological approaches, has not been well studied. Moreover, we show that expression of IMP3 mRNA was significantly overexpressed in SCC cell lines compared with TFM and KA. We found that IMP3 was expressed not only in the internal root sheath, but also in the granular layer of normal skin. To 
A

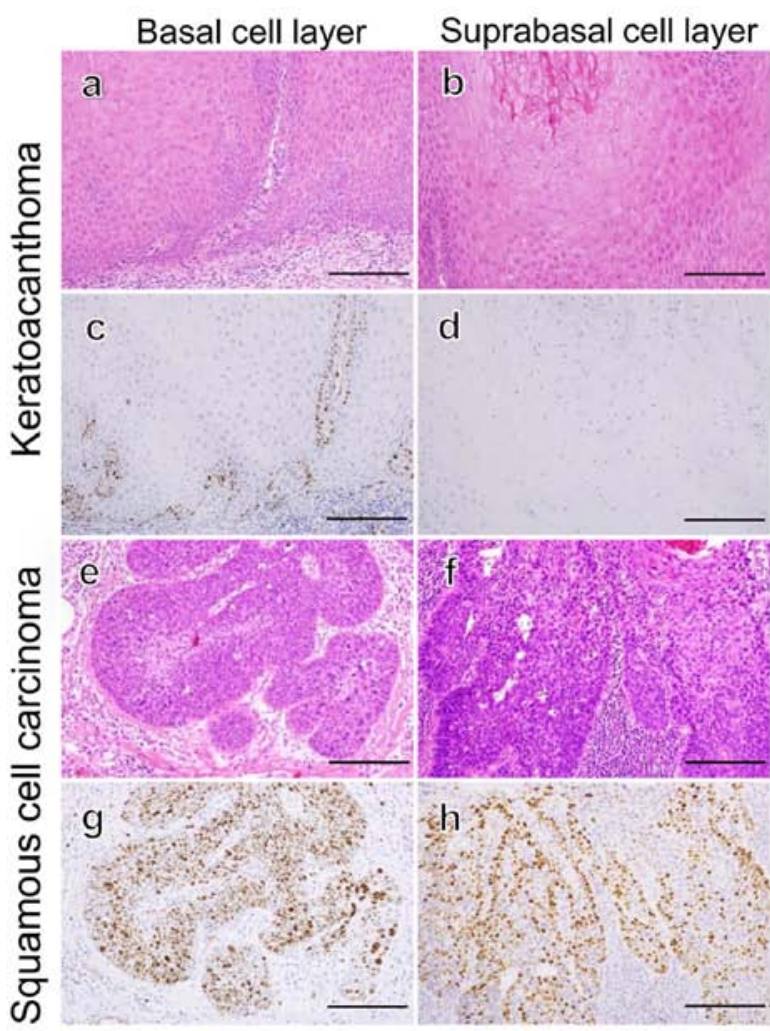

B

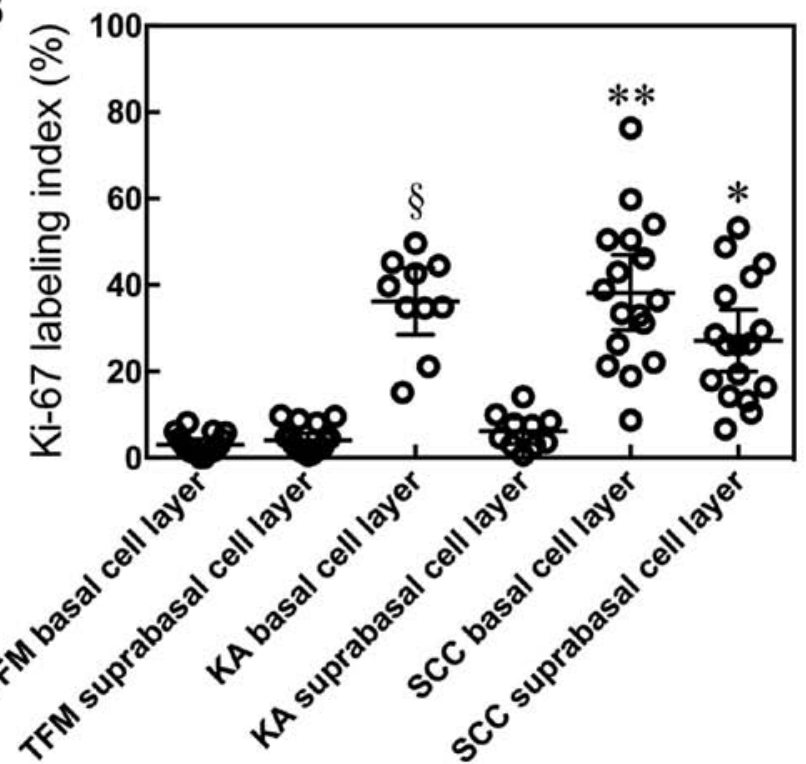

Figure 6. Histological and immunohistochemical staining of KA and SCC KA (a-d) and SCC (e-h) were examined by either a histological hematoxylin and eosin (H\&E) stain (a, b, e and $\mathrm{f}$ ) or immunohistochemical staining with anti-Ki-67 antibody (c, d, g and h) and peroxidase activity visualized by diaminobenzidine; sections were counterstained with Mayer's hematoxylin. $\mathrm{Ki}-67$ expression was localized in the basal cell layer of the tumor nest in $\mathrm{KA}$ (c and d) and in the whole layer of the tumor nest in SCC ( $\mathrm{g}$ and $\mathrm{h}$ ). Scale bars: $50 \mu \mathrm{m}$ (A). The Ki-67 labeling index was determined. Results are expressed as mean $\pm 95 \%$ confidence interval. ${ }^{*} \mathrm{P}<0.0001$ compared with the suprabasal cell layer of KA and TFM, Mann-Whitney U-test. ${ }^{* *} \mathrm{P}=0.0445$ compared with the suprabasal cell layer of $\mathrm{SCC},{ }^{* *} \mathrm{P}=0.8428$ compared with the basal cell layer of KA, Mann-Whitney U-test. ${ }^{\S} \mathrm{P}<0.0001$ compared with the suprabasal of KA and basal cell layer of TFM, Mann-Whitney U-test (B).

our knowledge, these results are the first to report on the role of IMP3 in SCC.
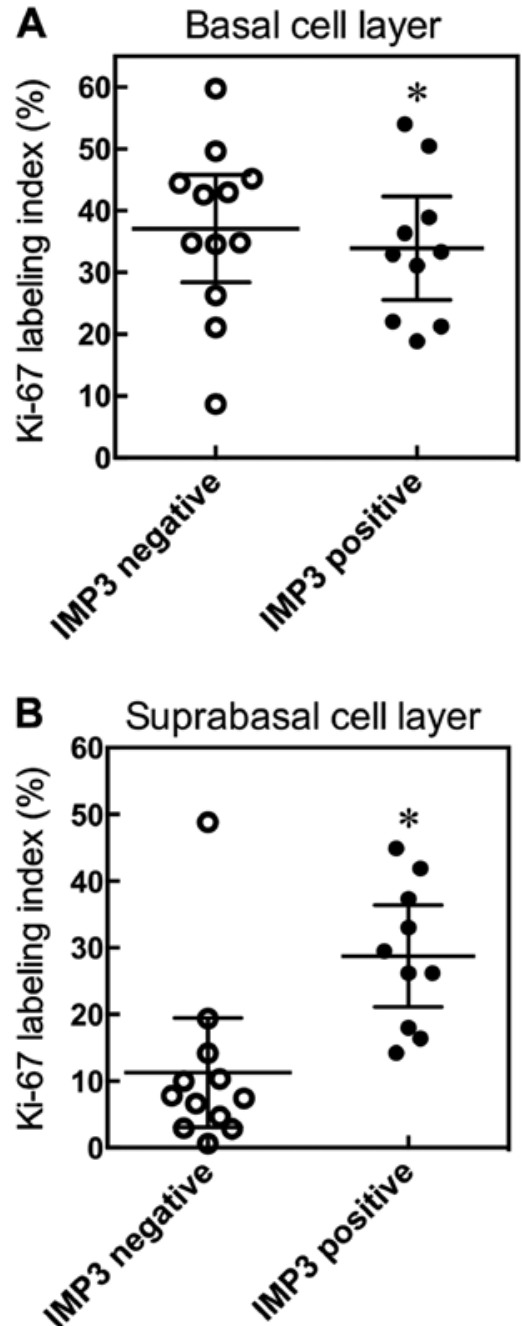

Figure 7. Relationship between Ki-67 and IMP3. SCC cases were divided into two groups, an IMP3 positive group and an IMP3 negative group, and the relationship between ki-67 and IMP3 in basal and suprabasal layers examined. Results are expressed as means $\pm 95 \%$ confidence interval. ${ }^{*} \mathrm{P}=0.4483$ compared with IMP3 negative group, Mann-Whitney U-test (A). * $\mathrm{P}=0.0022$ compared with IMP3 negative group, Mann-Whitney U-test (B).

Knockdown of IMP3 using siRNA reduced IMP3 mRNA and protein expression levels in the human SCC cell lines, HSC-1 and HSC-5, and in the immortalized human keratinocyte cell line, HaCaT. Moreover, knockdown of IMP3 reduced the proliferation of HSC-1 cells, and also significantly inhibited invasion by HSC-1 and HSC-5 cells. However, the knockdown of IMP3 in HaCaT cells did not influence cell invasion, in spite of the high level of IMP3 mRNA found in normal cells. These results raise the possibility that the kinetics of downstream signaling by IMP3 target genes differ in each cell line. With this in mind, we plan to undertake a detailed analysis of the cell signaling and target gene expression involved in IMP3 cell invasion in our next study. These data also suggest that IMP3 may promote cell invasion by cutaneous SCC cells, and may partially induce their cell proliferation. In this study, IMP3 expression did not correlate with clinicopathological variables such as patient age, gender, maximum diameter of tumors, ulceration, and tumor thickness in SCC.

Since all SCC cases were stage I or II without recurrent and metastatic lesions, our next study may focus on the late-stage 
cases, recurrent cases and metastasis cases. With regard to the relationship between IMP3 expression and the biological behavior of SCC from other organs, there are several reports suggesting that IMP3 expression might be associated with the lymph nodal and tumor stage of tumor invasion, as well as with overall survival in oral SCC $(30,31)$, also shown to be an independent prognostic factor in cervical SCC (32). Moreover, the high expression of IMP3 has been associated with lymph nodal metastasis and poor patient outcome in tongue SCC (33). Taken together, IMP3 may play an important role in the biological behavior of cutaneous SCC, and may be used as new therapeutic target for SCC.

KA was originally considered to be a benign neoplasm. However, a few cases of metastasis purported to originate from KAs have been reported, and many reports describe KA as a variant of SCC with low-grade malignancy $(3,34)$. Moreover, Sánchez Yus et al reported the first formal study on the 'transformation' of a subset of KAs into SCCs (35), and Weedon et al regarded KA as a benign lesion that could undergo malignant transformation into SCC (4). Weedon et al described how KA differed markedly from SCC in morphology, biological behavior, and outcome when perineural and/or intravenous invasion were present, and believed that KA was not a variant of SCC (36). Ni et al demonstrated that mRNA levels of mitogen-activated protein kinase 1 (MAPK1) and caspase-14 (CASP14) were upregulated in SCC, while those of BAG (Bcl-2associated athanogene) family molecular chaperone regulator 1 (BAG1) and matrix metalloproteinase-14 (MMP14) were downregulated in SCC, and suggested that SCC and KA were molecularly distinct entities (37). Recently, Soddu et al showed that IMP3 immunohistochemistry was useful in distinguishing KA from cutaneous SCC (24).

We demonstrated that IMP3 plays a role in cell proliferation and invasion in cutaneous SCC. In the histopathological diagnosis of a cutaneous tumor, distinguishing between SCC with a crateriform architecture and $\mathrm{KA}$ is often difficult; we suggest IMP3 may be a suitable marker. In our immunohistochemical study, IMP3 was observed in the internal root sheath and also in the granular layer of normal skin. Pryor et al also reported the expression of IMP3 in the internal root sheath (11). However, to our knowledge, this is the first report of the expression of IMP3 in the granular layer. Previous reports showed that IMP3 regulate CD44s expression in pancreatic cancer and hepatocellular carcinoma $(23,38)$. Liu et al found that the expression of syndecan, a cell surface proteoglycan like CD44, switched from the granular cell layer to the basal and lower spinous cell layers in irradiated mouse skin. They suggested that CD44 and syndecan play an important role in the proliferative process (39). Hyaluronan (HA) binds to CD44, and HA/CD44-mediated activation of RhoGTPase signaling leads to the regulation of keratinocyte activities and various epidermal functions, such as, proliferation, migration and differentiation (40). In our study, the tumor thickness of IMP3-positive SCC tended to be thicker than that of IMP3negative SCC (data not shown). Thus, the relationship between IMP3 and CD44-related molecules may be our next focus.

$\mathrm{Ki}-67$ is a cell cycle-regulating protein that is expressed during all phases of the cell cycle except G0. The cellular expression of $\mathrm{Ki}-67$ provides a measure of the growth fraction of a tumor (41). We demonstrated that the Ki-67 LI of SCC was significantly higher than that of KA, as reported previously by others $(42,43)$. However, a comparison between basal cell and suprabasal cell layers in Ki-67 LI analysis had not yet been made prior to this study. We demonstrate that the Ki-67 LI in the suprabasal cell layer of SCC was significantly higher than that in the suprabasal cell layer of KA, which indicates that the proliferative potential of suprabasal cells of SCC is higher than those of KA. We also found that IMP3-positive cells in the suprabasal cell layer tended to have a high Ki-67 LI. These are novel findings. IMP3 regulates the expression of cyclines D1, D3 and G1 (44). Lin et al demonstrated that the Ki-67 LI was significantly correlated with IMP3 expression in colon cancer (45). Similar to these findings, IMP3 may play an important role in the cell cycle of cutaneous SCC.

Other groups have described the mechanism of cell proliferation and invasion of IMP3 expression in cells. Recent studies have revealed that IMP3 promoted the progression of melanoma by regulating high mobility group AT-hook 2 (HMGA2) (18), the cell migration of renal cell carcinoma by activation of the NF- $\mathrm{NB}$ pathway (28), cell invasion and migration after epithelial to mesenchymal transition (EMT) (29), and matrix adhesion, cell motility and invasion of pancreatic carcinoma by enhancing CD44 and the transcription of kinesin KIF11 expression (23).

In conclusion, IMP3 may play an important role in the biological behavior of cutaneous SCC, and may be used as new therapeutic target for SCC. Moreover, the usefulness of IMP3 as a marker for distinguishing between SCC and KA warrants further evaluation.

\section{Acknowledgements}

This study was supported, in part, by grants-in-aid for the Clinical Rebiopsy Bank Project for Comprehensive Cancer Therapy Development to Z.N. from the Ministry of Education, Culture, Sport, Science and Technology, Japan (MEXT), 2013-2017 (S13110022) and a grant-in-aid for scientific research (C, no. 25461716 to S.A.) from JSPS KAKENHI.

\section{References}

1. Schwartz RA: The keratoacanthoma: A review. J Surg Oncol 12: 305-317, 1979.

2. Misago N, Inoue T, Koba S and Narisawa Y: Keratoacanthoma and other types of squamous cell carcinoma with crateriform architecture: Classification and identification. J Dermatol 40: 443-452, 2013.

3. Hodak E, Jones RE and Ackerman AB: Solitary keratoacanthoma is a squamous-cell carcinoma: Three examples with metastases. Am J Dermatopathol 15: 332-342, discussion 343-352, 1993.

4. Weedon DD, Malo J, Brooks D and Williamson R: Squamous cell carcinoma arising in keratoacanthoma: A neglected phenomenon in the elderly. Am J Dermatopathol 32: 423-426, 2010.

5. Kossard S, Tan KB and Choy C: Keratoacanthoma and infundibulocystic squamous cell carcinoma. Am J Dermatopathol 30: 127-134, 2008.

6. Misago N, Inoue $\mathrm{T}$, Toda $\mathrm{S}$ and Narisawa $\mathrm{Y}$ : Infundibular (follicular) and infundibulocystic squamous cell carcinoma: A clinicopathological and immunohistochemical study. Am J Dermatopathol 33: 687-694, 2011.

7. Müeller-Pillasch F, Lacher U, Wallrapp C, Micha A, Zimmerhackl F, Hameister H, Varga G, Friess H, Büchler M, Beger HG, et al: Cloning of a gene highly overexpressed in cancer coding for a novel KH-domain containing protein. Oncogene 14: 2729-2733, 1997. 
8. Nielsen J, Christiansen J, Lykke-Andersen J, Johnsen AH, Wewer UM and Nielsen FC: A family of insulin-like growth factor II mRNA-binding proteins represses translation in late development. Mol Cell Biol 19: 1262-1270, 1999.

9. Simon R, Bourne PA, Yang Q, Spaulding BO, di Sant'Agnese PA, Wang $\mathrm{HL}$ and $\mathrm{Xu} \mathrm{H}$ : Extrapulmonary small cell carcinomas express $\mathrm{K}$ homology domain containing protein overexpressed in cancer, but carcinoid tumors do not. Hum Pathol 38: 1178-1183, 2007.

10. Hammer NA, Hansen T, Byskov AG, Rajpert-De Meyts E, Grøndahl ML, Bredkjaer HE, Wewer UM, Christiansen J and Nielsen FC: Expression of IGF-II mRNA-binding proteins (IMPs) in gonads and testicular cancer. Reproduction 130: 203-212, 2005

11. Pryor JG, Simon RA, Bourne PA, Spaulding BO, Scott GA and $\mathrm{Xu} \mathrm{H}$ : Merkel cell carcinoma expresses $\mathrm{K}$ homology domain-containing protein overexpressed in cancer similar to other high-grade neuroendocrine carcinomas. Hum Pathol 40: 238-243, 2009.

12. Righi A, Zhang S, Jin L, Scheithauer BW, Kovacs K, Kovacs G, Goth MI, Korbonits M and Lloyd RV: Analysis of IMP3 expression in normal and neoplastic human pituitary tissues Endocr Pathol 21: 25-31, 2010.

13. King RL, Pasha T, Roullet MR, Zhang PJ and Bagg A: IMP-3 is differentially expressed in normal and neoplastic lymphoid tissue. Hum Pathol 40: 1699-1705, 2009.

14. Liao B, Hu Y, Herrick DJ and Brewer G: The RNA-binding protein IMP-3 is a translational activator of insulin-like growth factor II leader-3 mRNA during proliferation of human K562 leukemia cells. J Biol Chem 280: 18517-18524, 2005.

15. Clauditz TS, Wang CJ, Gontarewicz A, Blessmann M, Tennstedt $P$, Borgmann K, Tribius S, Sauter G, Dalchow C, Knecht R, et al: Expression of insulin-like growth factor II mRNA-binding protein 3 in squamous cell carcinomas of the head and neck. J Oral Pathol Med 42: 125-132, 2013.

16. Wang T, Fan L, Watanabe Y, McNeill PD, Moulton GG, Bangur C, Fanger GR, Okada M, Inoue Y, Persing DH, et al: L523S, an RNA-binding protein as a potential therapeutic target for lung cancer. Br J Cancer 88: 887-894, 2003.

17. Pryor JG, Bourne PA, Yang Q, Spaulding BO, Scott GA and Xu H: IMP-3 is a novel progression marker in malignant melanoma. Mod Pathol 21: 431-437, 2008

18. Sheen YS, Liao YH, Lin MH, Chu CY, Ho BY, Hsieh MC, Chen PC, Cha ST, Jeng YM, Chang CC, et al: IMP-3 promotes migration and invasion of melanoma cells by modulating the expression of HMGA2 and predicts poor prognosis in melanoma. J Invest Dermatol 135: 1065-1073, 2015.

19. Yu L, Xu H, Wasco MJ, Bourne PA and Ma L: IMP-3 expression in melanocytic lesions. J Cutan Pathol 37: 316-322, 2010.

20. Mentrikoski MJ, Ma L, Pryor JG, McMahon LA, Yang Q, Spaulding BO, Scott GA, Wang HL and Xu H: Diagnostic utility of IMP3 in segregating metastatic melanoma from benign nevi in lymph nodes. Mod Pathol 22: 1582-1587, 2009.

21. Takata A, Takiguchi S, Okada K, Takahashi T, Kurokawa Y, Yamasaki M, Miyata H, Nakajima K, Mori M and Doki Y: Expression of insulin-like growth factor-II mRNA-binding protein-3 as a marker for predicting clinical outcome in patients with esophageal squamous cell carcinoma. Oncol Lett 8 : 2027-2031, 2014

22. Zhang J, Ou Y, Ma Y, Zheng L, Zhang X, Xia R, Kong F, Shen Y, Wang S and Lin L: Clinical implications of insulin-like growth factor II mRNA-binding protein 3 expression in non-small cell lung carcinoma. Oncol Lett 9: 1927-1933, 2015.

23. Pasiliao CC, Chang CW, Sutherland BW, Valdez SM, Schaeffer D, Yapp DT and Ng SS: The involvement of insulin-like growth factor 2 binding protein 3 (IMP3) in pancreatic cancer cell migration, invasion, and adhesion. BMC Cancer 15: 266, 2015.

24. Soddu S, Di Felice E, Cabras S, Castellanos ME, Atzori L, Faa $\mathrm{G}$ and Pilloni L: IMP-3 expression in keratoacanthomas and squamous cell carcinomas of the skin: an immunohistochemical study. Eur J Histochem 57: e6, 2013.

25. Kondo S, Hozumi Y and Aso K: Autocrine secretion of an EGF-like substance by a cell line (HSC-1) derived from a human skin squamous cell carcinoma. J Dermatol Sci 2: 161-165, 1991.
26. Hozumi Y, Kondo S, Shimoura T and Aso K: Human squamous cell carcinoma from skin: Establishment and characterization of a new cell line (HSC-5). J Dermatol 17: 143-148, 1990.

27. Abdou AG, Maraee AH, El-Sayed EM and Elnaidany NF: Immunohistochemical expression of ezrin in cutaneous basal and squamous cell carcinomas. Ann Diagn Pathol 15: 394-401, 2011.

28. Pei X, Li M, Zhan J, Yu Y, Wei X, Guan L, Aydin H, Elson P, Zhou M, He H, et al: Enhanced IMP3 expression activates $\mathrm{NF}-\kappa \mathrm{B}$ pathway and promotes renal cell carcinoma progression. PLoS One 10: e0124338, 2015.

29. Su P, Hu J, Zhang H, Li W, Jia M, Zhang X, Wu X, Cheng H, Xiang L and Zhou G: IMP3 expression is associated with epithelial-mesenchymal transition in breast cancer. Int J Clin Exp Pathol 7: 3008-3017, 2014.

30. Lin CY, Chen ST, Jeng YM, Yeh CC, Chou HY, Deng YT, Chang CC and Kuo MY: Insulin-like growth factor II mRNAbinding protein 3 expression promotes tumor formation and invasion and predicts poor prognosis in oral squamous cell carcinoma. J Oral Pathol Med 40: 699-705, 2011.

31. Kim KY, Li S, Cha JD, Zhang X and Cha IH: Significance of molecular markers in survival prediction of oral squamous cell carcinoma. Head Neck 34: 929-936, 2012.

32. Wei Q, Yan J, Fu B, Liu J, Zhong L, Yang Q and Zhao T: IMP3 expression is associated with poor survival in cervical squamous cell carcinoma. Hum Pathol 45: 2218-2224, 2014

33. Li HG, Han JJ, Huang ZQ, Wang L, Chen WL and Shen XM: IMP3 is a novel biomarker to predict metastasis and prognosis of tongue squamous cell carcinoma. J Craniofac Surg 22: 2022-2025, 2011.

34. Schwartz RA: Keratoacanthoma: A clinico-pathologic enigma. Dermatol Surg 30: 326-333, 2004.

35. Sánchez Yus E, Simón P, Requena L, Ambrojo $P$ and de Eusebio E: Solitary keratoacanthoma: A self-healing proliferation that frequently becomes malignant. Am J Dermatopathol 22: 305-310, 2000

36. Weedon D, Malo J, Brooks D and Williamson R: Keratoacanthoma: Is it really a variant of squamous cell carcinoma? ANZ J Surg 80: 129-130, 2010.

37. Ni C, Su A, Ra S, Li X, Cochran A and Binder S: Squamous cell carcinoma arising from keratoacanthoma: An evaluation by RT-PCR. Am J Dermatopathol: Jun 2, 2015 (Epub ahead of print).

38. Hu S, Wu X, Zhou B, Xu Z, Qin J, Lu H, Lv L, Gao Y, Deng L, Yin J, et al: IMP3 combined with CD44s, a novel predictor for prognosis of patients with hepatocellular carcinoma. J Cancer Res Clin Oncol 140: 883-893, 2014.

39. Liu K, Kasper M, Bierhaus A, Langer S, Peterson I, Müller M and Trott KR: Differential expression of CD44s and CD44v10 proteins and syndecan in normal and irradiated mouse epidermis. Histochem Cell Biol 107: 159-167, 1997.

40. Bourguignon LY: Matrix hyaluronan-activated CD44 signaling promotes keratinocyte activities and improves abnormal epidermal functions. Am J Pathol 184: 1912-1919, 2014.

41. Brown DC and Gatter KC: Ki67 protein: The immaculate deception? Histopathology 40: 2-11, 2002.

42. Ribeiro D, Narikawa S and Marques ME: Expression of apoptotic and cell proliferation regulatory proteins in keratoacanthomas and squamous cell carcinomas of the skin. Pathol Res Pract 204: 97-104, 2008

43. Scola N, Segert HM, Stücker M, Altmeyer P, Gambichler T and Kreuter A: Ki-67 may be useful in differentiating between keratoacanthoma and cutaneous squamous cell carcinoma. Clin Exp Dermatol 39: 216-218, 2014

44. Rivera Vargas T, Boudoukha S, Simon A, Souidi M, Cuvellier S, Pinna G and Polesskaya A: Post-transcriptional regulation of cyclins D1, D3 and G1 and proliferation of human cancer cells depend on IMP-3 nuclear localization. Oncogene 33: 2866-2875, 2014.

45. Lin L, Zhang J, Wang Y, Ju W, Ma Y, Li L and Chen L: Insulin-like growth factor-II mRNA-binding protein 3 predicts a poor prognosis for colorectal adenocarcinoma. Oncol Lett 6: $740-744,2013$ 